\title{
Percutaneous Closure of Congenital Aortic-to-Right Atrial Fistula
}

\author{
Abdurrahman Akyüz ${ }^{1}$, Murat Çap² ${ }^{2}$ Ferhat Işık ${ }^{1}$, and Burhan Aslan ${ }^{1}$ \\ ${ }^{1}$ University of Health Sciences Diyarbakır Gazi Yaşargil Education and Research Hospital \\ ${ }^{2}$ Diyarbakir Gazi Yasargil Training and Research Hospital
}

September 24, 2021

\begin{abstract}
Transthoracic and transesophageal imaging of the 30-year-old patient who presented with palpitations and shortness of breath revealed flow from the aorta to the right atrium. Aortic-right atrial fistula, which is a rare anomaly, was evaluated. It was considered congenital, as there was no acquired cause. Since the patient was symptomatic, it was closed percutaneously with the Amplatzer Duct Occluder II device.
\end{abstract}

\section{INTRODUCTION}

Aortic-to-right atrial fistula (ARAF) is a rare anomaly. It can be congenital or acquired. Patients may present with dyspnea, chest pain, palpitations, fatigue or cough as local symptoms, fatigue and edema as systemic symptoms. Closure of symptomatic ARAF is recommended (1). It can be closed surgically or percutaneously.

\section{CASE PRESENTATION}

A 30-year-old male patient was admitted to our institution with a history of exertional dyspnea and palpitation for the last 4 months. On physical examination, there was a continuous murmur heard best along the right upper sternal border. A flow from the aorta to the right atrium was observed in the transthoracic echocardiography (TTE) (Fig. 1A,1B). The aortic valve was rheumatic and there was mild to moderate aortic regurgitation. Transesophageal echocardiography (TEE) and 3D TEE performed for additional information and revealed a shunt with a diameter of 5.5 millimeters from the noncoronary sinus valsalva to the right atrium (Video1; Fig. 2A, 2B). No acquired cause (infective endocarditis, aortic or cardiac operation, etc.) was found in the patient's history. Therefore, it was considered as congenital ARAF. It was decided to close because the patient had symptoms. The right femoral artery was cannulated using a 6-F sheath, and a fistula was visualized in the aortography (Fig. 3A). The fistula was cannulated with a 6-F Judkins right coronary catheter, and a 0.035 inch- $260 \mathrm{~cm}$ long hydrophilic guidewire was advanced over the catheter from the right atrium to the left femoral vein. The left femoral vein was cannulated with a 6 -F sheath, and an arteriovenous wire loop was created by taking it out of the left femoral vein through a guidewire snare. The Amplatzer Duct Occluder II (St. Jude Medical, St. Paul, Minnesota, USA) device with the delivery system from the left femoral vein was advanced through the sheath and inserted into the ARAF . There were no complications related to the procedure. After the device was released, angiography of the aortic root showed that the tunnel was completely closed(Video 2; Fig. 3B). After the closure procedure, imaging with TTE and 3D TTE showed that there was no residual flow and the device was in place (Fig. 4A, 4B). The symptoms of the patient decreased significantly 1 month after the procedure.

\section{DISCUSSION}


In this case, we presented a patient with congenital aortic-right atrium fistula that we detected in adulthood and which we closed percutaneously. According to our literature review, it is the second ARAF case that is congenital and detected in adulthood and closed percutaneously. The first case of ARAF was successfully closed by Patra S. et al (2) at the age of 22 with ARAF Amplatzer Vascular Plug II (St. Jude Medical, St. Paul, Minnesota, USA) that opened the aorta from the right coronary sinus through a giant aneurysm chamber into the right atrium.

The presence of blood flow between the aorta and atrium is a rare but complex pathological condition also known as aortic-atrial fistula (AAF). AAF can be congenital or acquired (infective endocarditis, trauma, aortic dissection, a complication of cardiac surgery, etc.). The exact incidence is currently unknown as AAF has not been extensively studied. There is also a lack of information regarding various aspects of AAF such as diagnostic strategies and management options. The embryological background and cause of congenital ARAF are unclear. Gajjar et al. (3) argue that the probable cause of this condition is a congenital deficiency of the elastic lamina in the media layer of the aorta, and as a result, due to high aortic pressure, the defective area in the aortic wall creates an extracardiac tunnel. It causes gradual enlargement and rupture of the right atrium due to the anatomical proximity and low filling pressure.

In the systematic review conducted by Jainandunsing JS et al.(1), a literature review was conducted and 136 AAF patients, including all age groups, were examined. ARAF was the most common with $63.2 \%$. The incidence of congenital ARAF was 9.6\%. ARAF was closed surgically in $73.5 \%$ and percutaneously in $10.3 \%$. Amplatzer device was used in $71.4 \%$ of percutaneous closures. As a treatment approach in this review; If small ARAFs are asymptomatic, close monitoring with diuretic therapy and active closure of the fistula should be considered if the clinical condition worsens. Large ARAF requires emergency closure by a percutaneous or surgical approach. Spontaneous closure of ARAF is very rare and conservative treatment should not be recommended in cases with large fistulas or clinical symptoms. Surgery and percutaneous closure had similar results.

In our case, closure was decided because the patient was symptomatic. Percutaneous intervention was preferred to surgery, as the fistula has a distinct origin and a narrow terminal ending in the right atrium.

\section{CONCLUSION}

Percutaneous closure can be considered as a well alternative to surgery in the treatment of congenital ARAF in adulthood because it is less invasive and has a shorter hospital stay.

\section{References}

1. Jainandunsing JS, Linnemann R, Bouma W, Natour N, Bidar E, Lorusso R, Gelsomino S, Johnson DM. Aorto-atrial fistula formation and closure: a systematic review. Natour E.J Thorac Dis. 2019 Mar;11(3):1031-1046. doi: 10.21037/jtd.2019.01.77.

2. Patra S, Mukhopadhyay S, Pande A, Chakraborty R, Chakrabarti N, Sarkar K. Pseudo-Cor Triatriatum Dextrum: A Rare Manifestation of Aorto-Right Atrial Fistula Successfully Closed by Amplatzer Vascular Plug. JACC Cardiovasc Interv. 2018 Apr 9;11(7):e51-e54. doi: 10.1016/j.jcin.2017.09.032. Epub 2018 Mar 14.

3. Gajjar T, Voleti C, Matta R, Iyer R, Dash PK, Desai N.J. Aorta-right atrial tunnel: clinical presentation, diagnostic criteria, and surgical options. Thorac Cardiovasc Surg. 2005 Nov;130(5):1287-92. doi: 10.1016/j.jtcvs.2005.07.021.

FIGURE 1. (A) Transthoracic echocardiography (TTE) apical 4-chamber view shows aorto-right atrium shunt (arrow) and (B) parasternal short axis view shows shunt from the aortic noncoronary sinus valsalva to the right atrium

FIGURE 2. (A and B) Transesophageal echocardiography (TEE) and 3D TEE image of the fistula(arrow)

FIGURE 3. (A) Angiographic image of aortic-to-right atrial fistula (arrow), (B) Angiographic image of the device after release 
FIGURE 4. (A and B) Transthoracic echocardiography(TTE) with no residual flow after percutaneous closure and 3D TTE view of the device

Video 1. Transesophageal echocardiography image of aortic-to-right atrial fistula

Video 2. Aortography image after device release

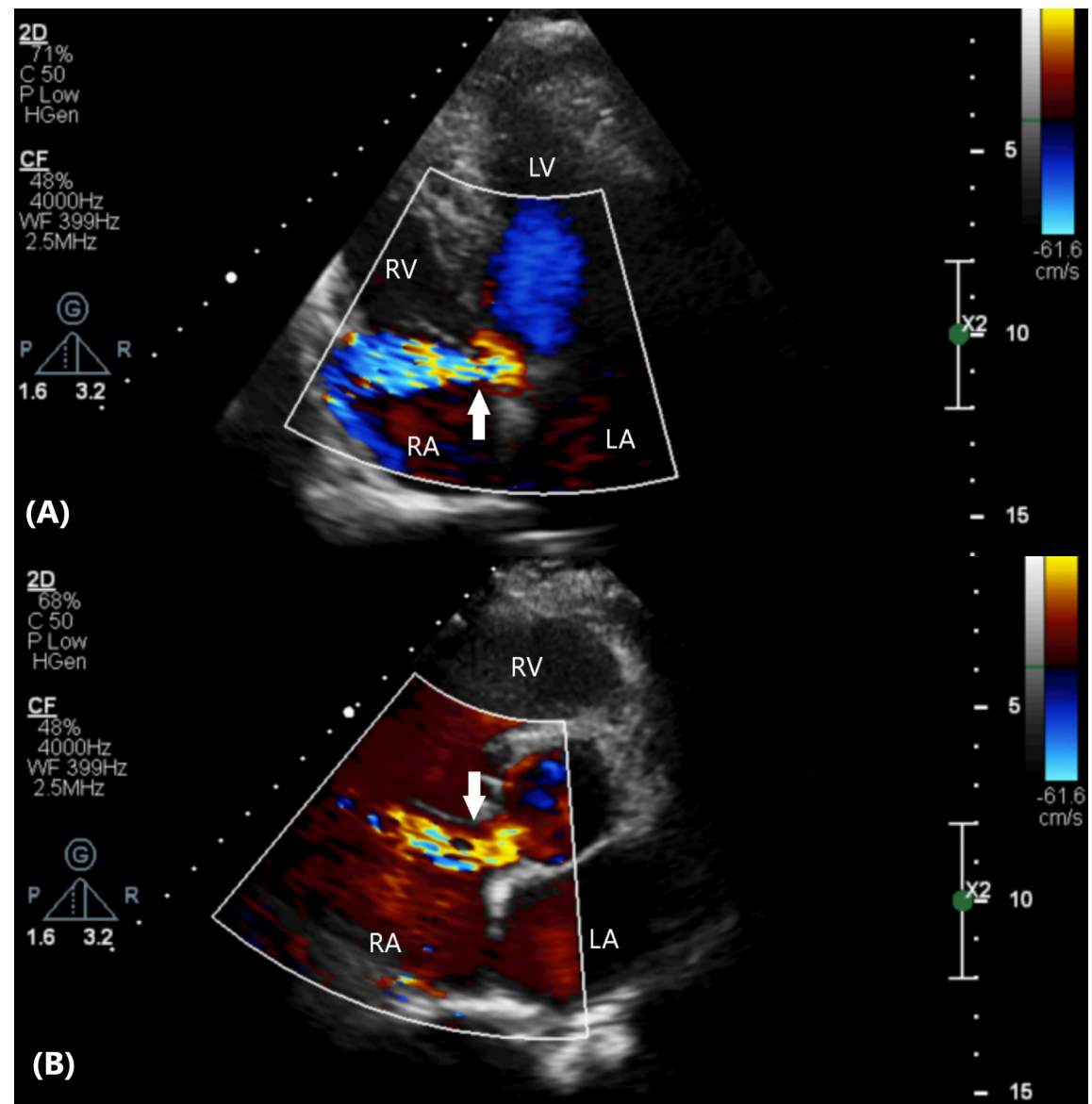




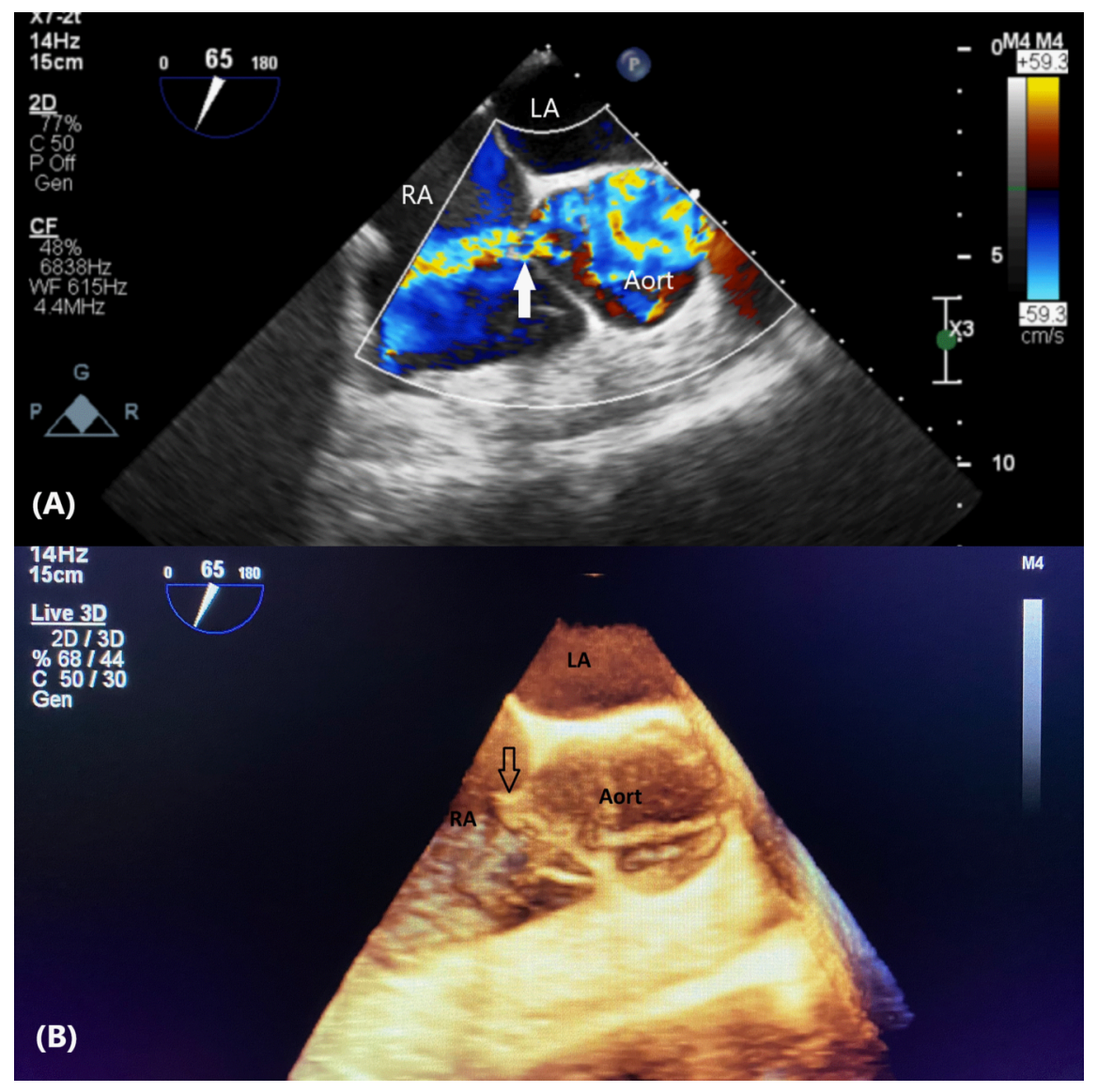




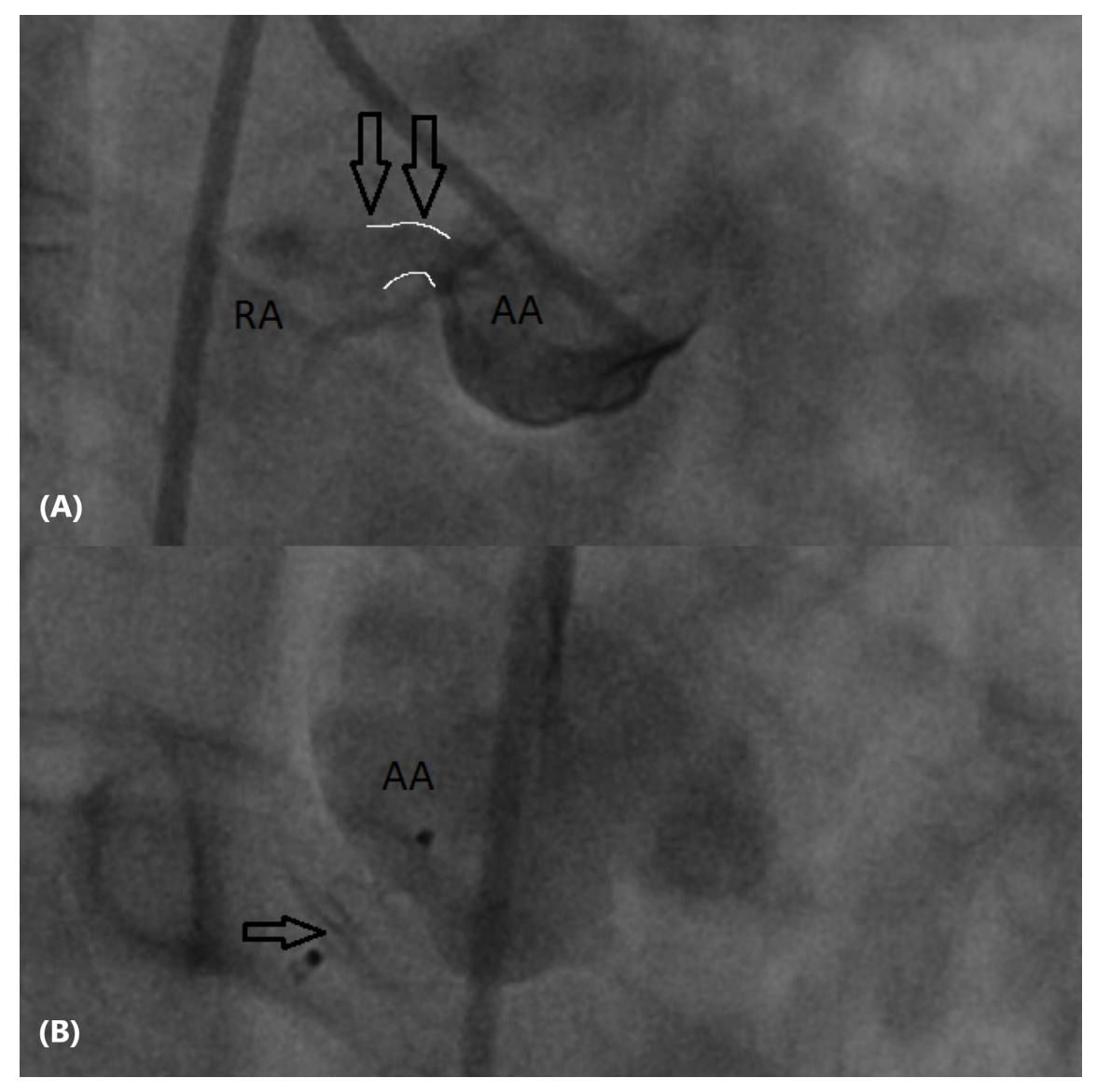




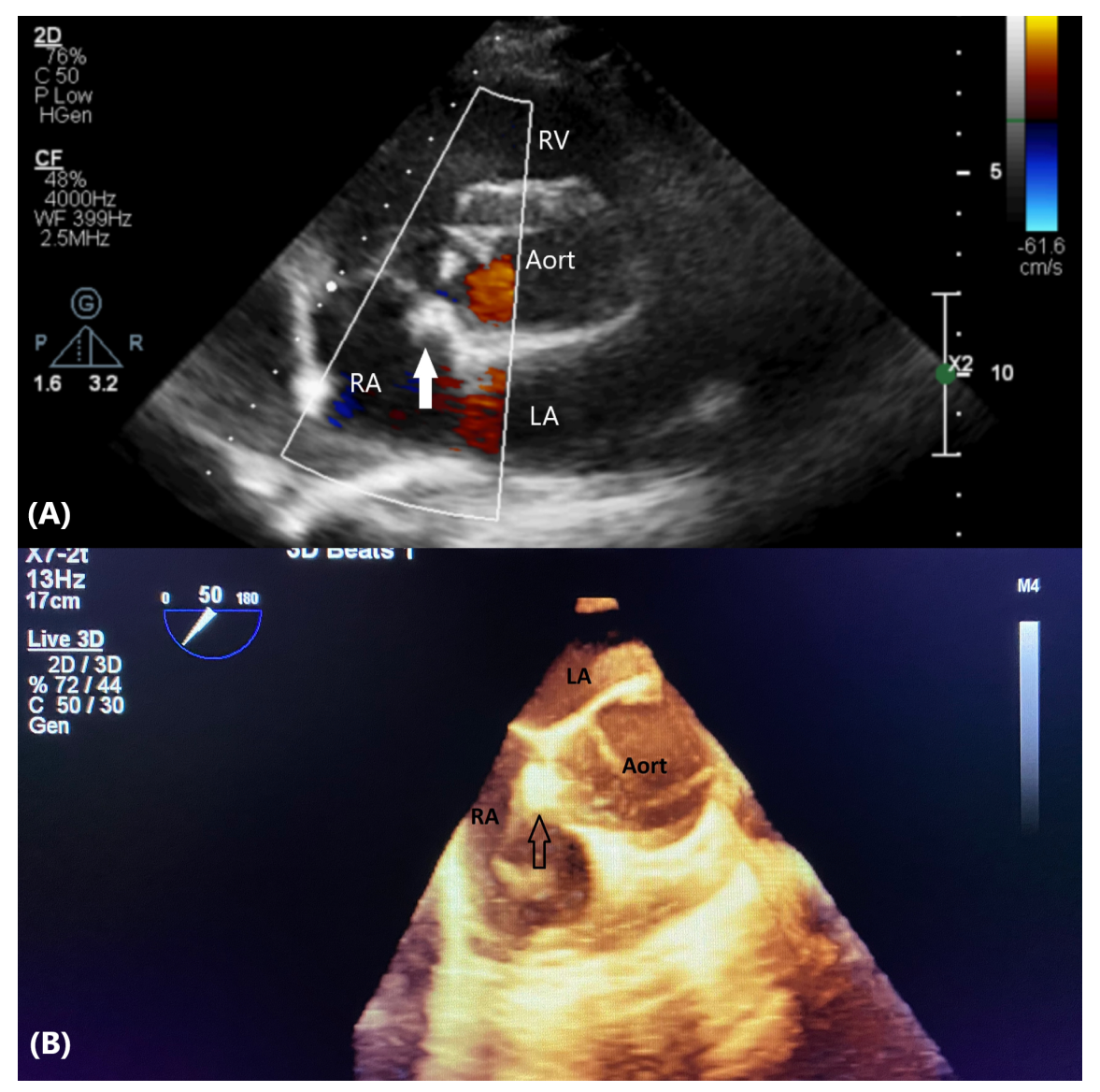

